\title{
Electrostatic ion waves in non-Maxwellian pair-ion plasmas
}

\author{
Kashif Arshad ${ }^{1,2}$ and S. Mahmood ${ }^{3,2}$ \\ ${ }^{1}$ Department of Physics, Quaid-i-Azam University, Islamabad 44000, Pakistan \\ ${ }^{2}$ National Centre for Physics, Quaid-i-Azam University Campus, Shadhra Valley Road, Islamabad 44000, \\ Pakistan \\ ${ }^{3}$ Theoretical Plasma Physics Division, PINSTECH, P.O. Nilore, Islamabad 44000, Pakistan
}

(Received 14 June 2010; accepted 2 November 2010; published online 7 December 2010)

\begin{abstract}
The electrostatic ion waves are studied for non-Maxwellian or Lorentzian distributed unmagnetized pair-ion plasmas. The Vlasov equation is solved and damping rates are calculated for electrostatic waves in Lorentzian pair-ion plasmas. The damping rates of the electrostatic ion waves are studied for the equal and different ion temperatures of pair-ion species. It is found that the Landau damping rate of the ion plasma wave is increased in Lorentzian plasmas in comparison with Maxwellian pair-ion plasmas. The numerical results are also presented for illustration by taking into account the parameters reported in fullerene pair-ion plasma experiments. (C) 2010 American Institute of Physics. [doi:10.1063/1.3520060]
\end{abstract}

During this decade, theoretical study of pair-ion (PI) plasma has gained attraction due to stable production of fullerene ion plasmas in the laboratory experiments. ${ }^{1-3}$ The dynamics of symmetric or PI plasma is different from the usual electron-ion plasma in which both fast and slow time scales occur due to difference in masses of ions and electrons. The collective behavior of fullerene PI plasmas has also been studied and three types of electrostatic waves, i.e., ion acoustic wave (IAW), intermediate frequency wave (IFW), and ion plasma wave (IPW) have been observed in the direction parallel to the magnetic field. The observed frequency ranges for IAW, IFW, and IPW are $\omega / 2 \pi$ $<12 \mathrm{kHz}, 12<\omega / 2 \pi<20 \mathrm{kHz}$, and $\omega / 2 \pi>20 \mathrm{kHz}$, respectively, and the ion cyclotron frequency is $\omega_{c} / 2 \pi$ $=4.3 \mathrm{kHz}$ at $B=0.2 \mathrm{~T}$. A lot of theoretical research work has already been published on linear and nonlinear electrostatic waves in PI plasmas. Mostly two fluid plasma theory is used to study the dynamics of PI plasmas. ${ }^{4-14}$ In PI plasmas, IPW and IAW can be derived from linear theory of two fluid plasma dynamics provided the temperature difference between the same mass ion species exist. In the experiments, it has been reported that a difference in temperature exists between two fullerene ion species which occurs due to different charging processes, i.e., the electron impact ionization and attachment for the production of both positive and negative of fullerene ions in PI plasmas. ${ }^{2}$ The third mode IFW cannot be obtained from linear theory using two fluid model in homogeneous PI plasmas ${ }^{2,7}$ and its theoretical understanding is still not clear. The surface ion waves in PI plasmas has been studied by Hasegawa and Shukla. ${ }^{4}$ Schamel and Luque ${ }^{15}$ investigated electrostatic waves in PI plasmas with the inclusion of trapped ions in the potential troughs in their model. Recently, the arbitrary amplitude solitary waves in PI plasma have been study by Dubinov et al. ${ }^{8}$ They studied that electrostatic solitary waves are formed only when there exist a small difference of temperatures between PI species. The low amplitude solitons and shocks have been studied in PI plasma using reductive perturbation method. ${ }^{10-12}$ It has been reported the nonlinear compressive and rarefactive electro- static structures are formed only when a little difference of temperature occurs between the PI species. Recently, a criterion for pure PI has been studied for its production in laboratory experiments. ${ }^{16}$

The electrostatic modes parallel to magnetic field in PI plasmas have been studied by Vranjes and Poedts ${ }^{17}$ for same temperature PI species. They studied the resonant damping of longitudinal electrostatic waves in PI plasma using the kinetic model for Maxwellian distributed PI plasmas. However, it has been mentioned by the authors that the choice of Maxwellian plasmas in their study is not a good assumption because ions collected from the exciter hole may not have completely followed the Maxwellian distribution in PI (fullerene) plasma experiment. ${ }^{2}$ The non-Maxwellian velocity distributed plasmas have been observed in space and astrophysical plasma situations. The observed particles are found to have distribution of quasi-Maxwellian up to mean thermal velocities with non-Maxwellian suprathermal tails at high velocities and energies. ${ }^{18}$ The nonthermal plasmas are found to exist in the magnetospheres of the Earth and in planets and also in the solar wind. ${ }^{19-21}$ In general, the observed non-Maxwellian plasma distributed particles are well fitted with the generalized Lorentzian or kappa distribution, which contains both thermal as well as suprathermal parts of the observed velocity spectra. The kappa distributions have been used by a number of authors to study the damping rates of the electrostatic and electromagnetic waves in plasmas. Therefore, it will be interesting to study longitudinal waves in PI plasma with kappa distribution function, so that the PI species are assumed to be nonthermal in the laboratory experiment. The kappa distribution function approaches to Maxwellian when the spectral index kappa approaches to infinity. Recently, the damping rate for Langmuir, dust ion acoustic, and dust acoustic waves are studied in generalized Lorentzian multicomponent plasmas. $^{22,23}$

In this brief communication, we study the electrostatic longitudinal waves in the presence of non-Maxwellian distributed (generalized Lorentzian or kappa distribution) pure PI plasma containing positive and negative fullerene $\left(\mathrm{C}_{60}^{ \pm}\right)$ 
ions. The Vlasov equation for the $\alpha$ species is given by

$$
\frac{\partial f_{\alpha}}{\partial t}+\mathbf{v} \cdot \nabla f_{\alpha}+\mathbf{a} \cdot \nabla_{v} f_{\alpha}=0,
$$

where $\mathbf{a}=\mathbf{q}_{\alpha}(\mathbf{E}+\mathbf{v} \times \mathbf{B}) / m_{\alpha}$ and $\alpha=+,-$ for the positive and negative ions, respectively. Let us suppose that we have no macroscopic field $\mathbf{E}_{0}=0$ and $\mathbf{B}_{0}=0$ and the PI plasma is assumed to be unmagnetized or assuming the plasma dynamics parallel to the magnetic field. The perturbed linear distribution function is obtained as follows:

$$
f_{\alpha 1}=-\frac{q_{\alpha}}{i m_{\alpha}} \frac{\mathbf{E}_{1} \cdot \nabla_{u} f_{\alpha 0}}{\left(\omega-k v_{\|}\right)} .
$$

The value of the plasma current can be calculated from the expression $\mathbf{J}_{\alpha}=\Sigma_{\alpha} q_{\alpha} n_{\alpha} \mathbf{v}_{\alpha}$ which is used in order to calculate the conductivity tensor. The conductivity tensor helps to calculate the dielectric function for above given plasma, where macroscopic density and velocity, i.e., $n_{\alpha}$ and $\mathbf{v}_{\alpha}$ for $\alpha$ species, are defined as $n_{\alpha}=\Sigma_{\alpha} \iiint f_{\alpha} d^{3} \mathbf{v}$ and $\mathbf{v}_{\alpha}$ $=\left(\iiint \mathbf{v} f_{\alpha} d^{3} \mathbf{v}\right) / n_{\alpha}$, respectively. Using the expressions of $n_{\alpha}$, $\mathbf{v}_{\alpha}$, and $f_{\alpha}$ in the plasma current density defined in the linear limit, i.e., $J_{r 1}=\sigma_{r s} E_{s 1}$, where $\sigma_{r s}$ is the conductivity tensor defined in plasmas and summation is over the repeated index, the conductivity tensor is given by

$$
\sigma_{r s}=-\sum_{\alpha} \frac{q_{\alpha}^{2}}{i m_{\alpha}} \iiint \frac{\mathbf{v}_{\alpha} \nabla_{v} f_{\alpha 0}}{\left(\omega-k v_{\|}\right)} d^{3} \mathbf{v} .
$$

Now choosing the three dimensional non-Maxwellian distribution function such as kappa or generalized Lorentzian distribution for PI species, which is given by ${ }^{19,20}$

$f_{\alpha 0}=\frac{1}{\pi^{3 / 2} \theta_{\perp \alpha}^{2} \theta_{\| \alpha}} \frac{\lceil(\kappa+1)}{\kappa^{3 / 2} \Gamma(\kappa-1 / 2)}\left[1+\frac{v_{\|}^{2}}{\kappa \theta_{\| \alpha}^{2}}+\frac{v_{\perp}^{2}}{\kappa \theta_{\perp \alpha}^{2}}\right]^{-\kappa-1}$,

where $\kappa$ is the spectral index of Lorentzian distribution having values $>3 / 2$ and $\Gamma$ is represented for Gamma function. The Lorentzian thermal speed is related to the particle temperature (in the energy units) by the relation $\theta_{\| \alpha, \perp \alpha}^{2}=(2 \kappa$ $-3 / \kappa) v_{T_{\| \omega^{\prime}} \perp \alpha}^{2}$ and thermal speeds of the ion species are de-

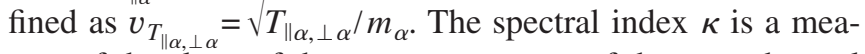
sure of the slope of the energy spectrum of the suprathermal particles in the tail of the velocity distribution function. ${ }^{18}$ The smaller the value of $\kappa$ the more suprathermal particles are in the distribution function tail and harder the energy spectrum. The kappa distribution approaches the Maxwellian as $\kappa \rightarrow \infty$. After using the kappa distribution function described above in Eq. (3) the conductivity tensor is obtained as follows:

$$
\begin{aligned}
\sigma_{r s}= & -\frac{i}{\pi^{3 / 2}} \frac{1}{4 \pi} \sum_{\alpha} \frac{\omega_{p \alpha}^{2}}{\theta_{\perp \alpha}^{2} \theta_{\| \alpha}^{3}} \frac{\lceil(\kappa+1)}{\kappa^{3 / 2}\lceil(\kappa-1 / 2)} \frac{(\kappa+1)}{\kappa} \\
& \times \iiint \frac{v_{r} v_{s}}{\left(\omega-k v_{\|}\right)}\left[1+\frac{v_{\|}^{2}}{\kappa \theta_{\| \alpha}^{2}}+\frac{v_{\perp}^{2}}{\kappa \theta_{\perp \alpha}^{2}}\right]^{-\kappa-2} d^{3} \mathbf{v},
\end{aligned}
$$

where $\omega_{p \alpha}=\sqrt{4 \pi n_{0 \alpha} q_{\alpha}^{2} / m_{\alpha}}$ is the plasma frequency of the $\alpha$ species and the $r, s$ in the subscript denote the coordinate axes of the given coordinate system. Now evaluating the integral in Eq. (5) and using the expressions given as follows:

$$
\int_{-\infty}^{\infty} v_{\|}^{2}\left[1+\frac{v_{\|}^{2}}{\kappa \theta_{\| \alpha}^{2}}\right]^{-\kappa-1} d v_{\|}=\frac{\sqrt{\pi}}{2} \theta_{\| \alpha}^{3} \frac{\kappa^{3 / 2}\lceil(\kappa-1 / 2)}{\lceil(\kappa+1)}
$$

and

$$
\int_{-\infty}^{\infty} v_{\|}^{4}\left[1+\frac{v_{\|}^{2}}{\kappa \theta_{\| \alpha}^{2}}\right]^{-\kappa-1} d v_{\|}=\frac{3 \sqrt{\pi}}{4} \theta_{\| \alpha}^{5} \frac{\kappa^{5 / 2}\lceil(\kappa-3 / 2)}{\lceil(\kappa+1)} .
$$

The dielectric function in the parallel direction is given by

$$
\epsilon_{\| \alpha}=1+\sum_{\alpha} \frac{\omega_{p \alpha}^{2}}{2 k^{2} \theta_{\| \alpha}^{2}}\left[1+Z\left(\frac{\omega}{k \theta_{\| \alpha}}\right)\right]
$$

where dielectric tensor $\epsilon_{r s}=\hat{\delta}+i 4 \pi \sigma_{r s} / \omega$ has been defined and $\hat{\delta}$ is the unitary tensor. The dispersion function in nonMaxwellian plasmas is defined as

$$
Z\left(\frac{\omega}{k \theta_{\| \alpha}}\right)=\frac{1}{\sqrt{2 \pi}}\left(\frac{\omega}{k \theta_{\| \alpha}}\right) \int_{-\infty}^{\infty} \frac{v_{\|}^{2}}{\left(\omega-k v_{\|}\right)}\left[1+\frac{v_{\|}^{2}}{\kappa \theta_{\| \alpha}^{2}}\right]^{-\kappa-1} d \mathbf{v}_{\|} .
$$

The above defined dispersion function can be expanded in the limits described as follows, i.e., for $\left|\omega /\left(k \theta_{\| \alpha}\right)\right| \ll 1$,

$$
Z\left(\frac{\omega}{k \theta_{\| \alpha}}\right)=-i \sqrt{\pi}\left(\frac{\omega}{k \theta_{\| \alpha}}\right) \frac{\lceil(\kappa+1)}{\kappa^{3 / 2}\lceil(\kappa-1 / 2)}\left[1+\frac{\omega^{2}}{\kappa k^{2} \theta_{\| \alpha}^{2}}\right]^{-\kappa-1},
$$

and for $\left|\omega /\left(k \theta_{\| \alpha}\right)\right| \gg 1$, we have

$$
\begin{aligned}
Z\left(\frac{\omega}{k \theta_{\| \alpha}}\right)= & +\left(\frac{k \theta_{\| \alpha}}{\omega}\right)^{2}+\frac{3 \kappa}{2(3 \kappa-2)}\left(\frac{k \theta_{\| \alpha}}{\omega}\right)^{4}+\cdots \\
& -i \sqrt{\pi}\left(\frac{\omega}{k \theta_{\| \alpha}}\right) \frac{\lceil(\kappa+1)}{\kappa^{3 / 2}\lceil(\kappa-1 / 2)}\left[1+\frac{\omega^{2}}{\kappa k^{2} \theta_{\| \alpha}^{2}}\right]^{-\kappa-1} .
\end{aligned}
$$

There is no wave propagation in the limit $\left|\omega /\left(k \theta_{\| \alpha}\right)\right| \ll 1$, and the high frequency longitudinal waves can exist only in the limit $\left|\omega /\left(k \theta_{\| \alpha}\right)\right| \gg 1$.

Therefore, the longitudinal waves in the limit $\left|\omega /\left(k \theta_{\| \alpha}\right)\right| \gg 1$ in nonthermal PI plasmas can be obtained through the dielectric function described in Eq. (6) in the parallel direction as follows:

$$
\begin{aligned}
\epsilon_{\| \alpha}= & 1-\sum_{\alpha} \frac{\omega_{p \alpha}^{2}}{2 \omega^{2}}\left[1+\frac{3 \kappa}{2(3 \kappa-2)} \frac{k^{2} \theta_{\| \alpha}^{2}}{\omega^{2}}\right] \\
& +i \sqrt{\pi} \frac{\omega_{p \alpha}^{2}}{\theta_{\| \alpha}^{3}} \frac{\omega}{k^{3}} \frac{\Gamma(\kappa+1)}{\kappa^{3 / 2}\lceil(\kappa-1 / 2)}\left[1+\frac{\omega^{2}}{\kappa k^{2} \theta_{\| \alpha}^{2}}\right]^{-\kappa-1}=0 .
\end{aligned}
$$

The angular frequency is described as $\omega=\omega_{r} \pm i \gamma$ for the slowly growing and decreasing mode with the assumption that the imaginary part of the dispersion relation is much smaller than the real part, i.e., $\left|\omega_{r}\right| \gg|\gamma|$. The dispersion relation of the ion plasma wave for a kappa distributed (or Lorentzian) PI plasma can be obtained from Eq. (7) described as follows:

$$
\omega_{r}^{2}=\frac{1}{2}\left(\omega_{p+}^{2}+\omega_{p-}^{2}\right)+\frac{3 \kappa}{(2 \kappa-3)} \frac{k^{2}}{\left(\omega_{p+}^{2}+\omega_{p-}^{2}\right)} v_{T_{\text {eff' }}}^{2}
$$

where effective thermal speed of the species is defined as $v_{T_{\text {eff }}}=\omega_{p+} \theta_{\|+}\left[1+\omega_{p-}^{2} \theta_{\|-}^{2} / \omega_{p+}^{2} \theta_{\|+}^{2}\right]^{1 / 2}$. 
The damping rate $\gamma$ of the ion longitudinal mode in PI plasma is obtained by using the definition $\gamma=-\epsilon_{i} /\left(\partial \epsilon_{r} / \partial \omega_{r}\right)$, which is given by

$$
\begin{aligned}
\gamma= & -\sqrt{\frac{\pi}{8}} \frac{\lceil(\kappa+1)}{(\kappa-3 / 2)^{3 / 2}\lceil(\kappa-1 / 2)} \frac{\left(\omega_{p+}^{2}+\omega_{p-}^{2}\right)}{k^{3}} \\
& \times\left[\frac{1}{\omega_{p+} \lambda_{D+}^{3}}\left(1+\frac{\omega^{2}}{\kappa k^{2} \theta_{\|+}^{2}}\right)^{-\kappa-1}\right. \\
& \left.+\frac{1}{\omega_{p-} \lambda_{D-}^{3}}\left(1+\frac{\omega^{2}}{\kappa k^{2} \theta_{\|-}^{2}}\right)^{-\kappa-1}\right] .
\end{aligned}
$$

From above equation, the damping rate of the ion longitudinal wave in Lorentzian unmagnetized PI plasmas can be calculated with variation of spectral index $\kappa$ and fixed temperature ratio of the PI species where Debye lengths of the PI species $\lambda_{D \alpha}=\sqrt{T_{\alpha} / 4 \pi n_{0 \alpha} q_{\alpha}^{2}}$ have been defined.

By using the value of real frequency in the damping factor given in Eq. (8), the equation takes the form

$$
\begin{aligned}
\gamma= & -\sqrt{\frac{\pi}{8}} \frac{\lceil(\kappa+1)}{(\kappa-3 / 2)^{3 / 2}\lceil(\kappa-1 / 2)} \frac{\left(\omega_{p+}^{2}+\omega_{p-}^{2}\right)}{k^{3}} \\
& \times\left[\frac { 1 } { \omega _ { p + } \lambda _ { D + } ^ { 3 } } \left\{1+\frac{1}{(2 \kappa-3) k^{2} \lambda_{D+}^{2}}\right.\right. \\
& \left.+\frac{3}{(2 \kappa-3) \theta_{\|+}^{2}}\left(\frac{\omega_{p+}^{2} \theta_{\|+}^{2}+\omega_{p-}^{2} \theta_{\|-}^{2}}{\left(\omega_{p+}^{2}+\omega_{p-}^{2}\right)}\right)\right\}^{-\kappa-1} \\
& +\frac{1}{\omega_{p-} \lambda_{D-}^{3}}\left\{1+\frac{1}{(2 \kappa-3) k^{2} \lambda_{D-}^{2}}\right. \\
& \left.\left.+\frac{3}{(2 \kappa-3) \theta_{\|-}^{2}}\left(\frac{\omega_{p+}^{2} \theta_{\|+}^{2}+\omega_{p-}^{2} \theta_{\|-}^{2}}{\left(\omega_{p+}^{2}+\omega_{p-}^{2}\right)}\right)\right\}^{-\kappa-1}\right] .
\end{aligned}
$$

For pure pair-ion plasma, we have mass and number density of both the species are equal (i.e., $m_{p}=m_{n}=m$ and $n_{0+}=n_{0-}$ $=n$ ), so we have $\omega_{p+}^{2}=\omega_{p-}^{2}$.

Therefore, when PI species have the same temperature $T_{+}=T_{-}$and the Debye lengths of both the species are the same, i.e., $\left(\lambda_{D_{+}}=\lambda_{D_{-}}\right)$. Then normalized damping rate of the ion plasma wave is obtained from Eq. (10) described as follows:

$$
\begin{aligned}
\frac{\gamma}{\omega_{p+}}= & -\sqrt{\frac{\pi}{8}} \frac{\lceil(\kappa+1)}{(\kappa-3 / 2)^{3 / 2}\lceil(\kappa-1 / 2)} \frac{2}{k^{3} \lambda_{D_{+}}^{3}} \\
& \times\left[1+\frac{1}{(2 \kappa-3) k^{2} \lambda_{D+}^{2}}+\frac{6}{(2 \kappa-3)}\right]^{-\kappa-1} .
\end{aligned}
$$

For the case, when PI species have different temperature $T_{+} \neq T_{-}$, so the Debye lengths of both the species are not equal $\left(\lambda_{D+} \neq \lambda_{D_{-}}\right)$. The normalized damping rate of the longitudinal waves in PI plasma obtained from Eq. (10) is given by

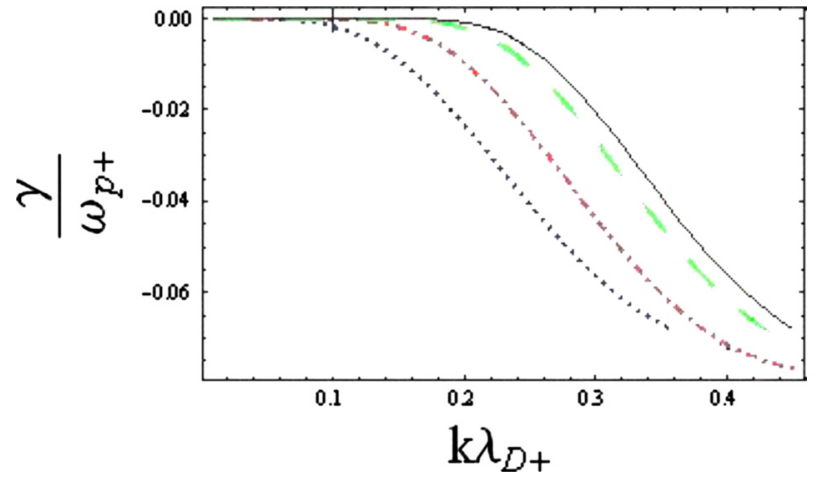

FIG. 1. (Color online) Normalized damping rates are plotted for electrostatic ion waves in PI plasmas for different spectral indices $\kappa=3$ (blue dotted curve), 5 (red dot-dashed curve), 10 (green dashed curve), and 15 (black solid curve) are shown at fixed ion temperature ratios $T_{+} / T_{-}=1$.

$$
\begin{aligned}
\frac{\gamma}{\omega_{p+}}= & -\sqrt{\frac{\pi}{8}} \frac{\lceil(\kappa+1)}{(\kappa-3 / 2)^{3 / 2}\lceil(\kappa-1 / 2)} \frac{1}{k^{3} \lambda_{D_{+}}^{3}} \\
& \times\left[\left\{1+\frac{1}{(2 \kappa-3) k^{2} \lambda_{D+}^{2}}+\frac{3}{(2 \kappa-3)}\left(1+\frac{T_{-}}{T_{+}}\right)\right\}^{-\kappa-1}\right. \\
& +\left(\frac{T_{+}}{T_{-}}\right)\left\{1+\left(\frac{T_{+}}{T_{-}}\right) \frac{1}{(2 \kappa-3) k^{2} \lambda_{D-}^{2}}\right. \\
& \left.\left.+\left(\frac{T_{+}}{T_{-}}\right) \frac{3}{(2 \kappa-3)}\left(1+\frac{T_{-}}{T_{+}}\right)\right\}^{-\kappa-1}\right] .
\end{aligned}
$$

We have plotted normalized damping rates of electrostatic ion waves for Lorentzian PI plasmas described in Eqs. (11) and (12). The typical parameters of ion temperatures and densities reported in PI plasmas experiment, i.e., $T_{+}=T_{-}$ $=0.3-0.5 \mathrm{eV}$ and $n_{0+}=n_{0-}=2 \times 10^{8} \mathrm{~cm}^{-3},{ }^{3}$ have been used for numerical analysis. The normalized damping rates are plotted against normalized wave number for different values of spectral index $\kappa$ of the generalized Lorentzian distribution function as shown in Fig. 1. It is found that damping rate of the electrostatic ion waves is increased for small values of $\kappa$ at fixed temperature ratio of positive to negative ions in PI plasmas. The physical explanation is that at small values of $\kappa$, there are more energetic ions in comparison of large $\kappa$

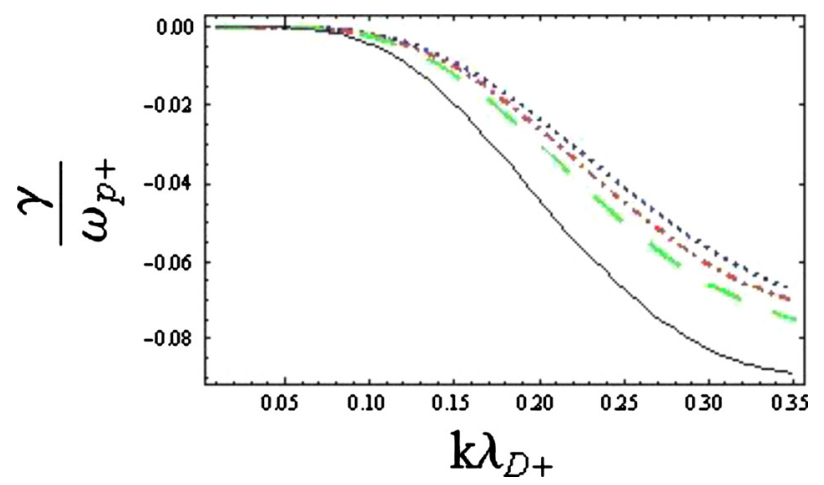

FIG. 2. (Color online) Normalized damping rates vs normalized wave numbers are shown for the case $T_{+} \leq T_{-}$at fixed value of $\kappa=3$. The damping curves correspond to $T_{+} / T_{-}=1$ (blue dotted curve), 0.9 (red dot-dashed curve), 0.8 (green dashed curve), and 0.6 (black solid curve), respectively. 


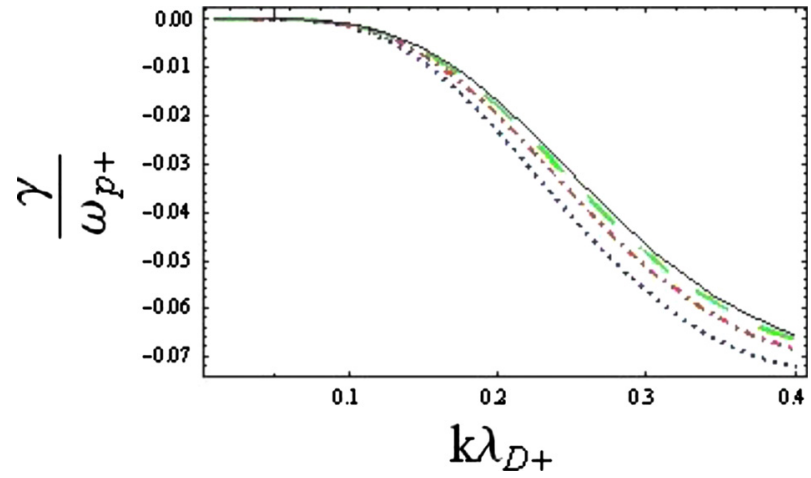

FIG. 3. (Color online) Normalized damping rates vs normalized wave numbers are shown for the case $T_{+} \geq T_{-}$at fixed value of $\kappa=3$. The damping curves correspond to $T_{+} / T_{-}=1$ (blue dotted curve), 1.2 (red dot-dashed curve), 1.4 (green dashed curve), and 1.6 (black solid curve), respectively.

value (i.e., Maxwellian distribution function) of the spectrum and the number of particles taking energy from the waves are increased and therefore the damping rate of the ion thermal wave is increased in Lorentzian PI plasmas in comparison with Maxwellian plasmas. In Fig. 2, the normalized damping rates are plotted in the limit $T_{+}<T_{-}$for the fixed low value of spectral index, i.e., $\kappa=3$. It can be seen from the figure that as the temperature ratio of the pair ions $T_{+} / T_{-}$is decreased, the damping rate of the electrostatic ion wave is decreased in comparison with the case of equal temperature of the ion species. Figure 3 shows that damping rate of the electrostatic ion wave is increased for the case $T_{+}>T_{-}$with the increase in the temperature ratio of the PI species in comparison with the temperature of same ion species in PI plasmas. The damping rates for Maxwellian PI plasma (i.e., for large $\kappa$ values) at different ion temperatures $T_{+}<T_{-}$and $T_{+}>T_{-}$and also for same temperature of the ion species are shown in Figs. 4 and 5, respectively. It can be seen from the figures that damping rate of the ion plasma waves in kappa distributed non-Maxwellian PI plasmas is large in comparison with the Maxwellian PI plasmas.

In conclusion, we have studied damping rates of electrostatic ion waves in pure pair-ion Lorentzian and Maxwellian distributed plasmas for same and different temperatures of

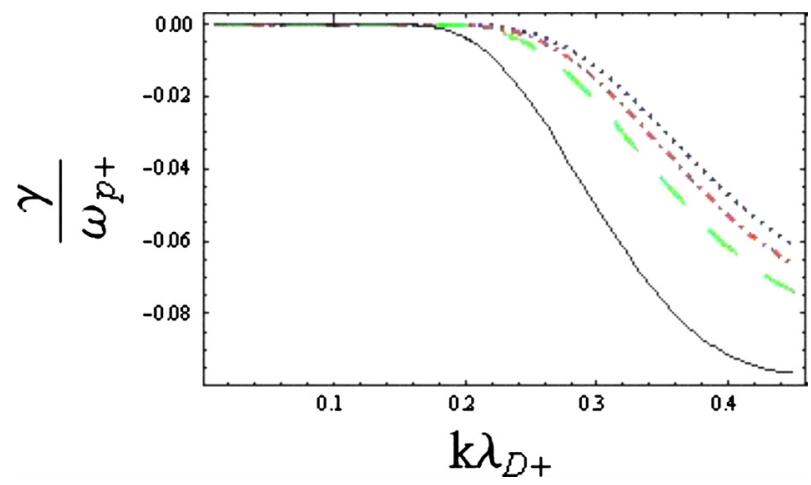

FIG. 4. (Color online) Normalized damping rates of electrostatic ion waves are shown for Maxwellian (i.e., $\kappa=50$ ) PI plasma for the case $T_{+} \leq T_{-}$, where damping curves correspond to $T_{+} / T_{-}=1$ (blue dotted curve), 0.9 (red dotdashed curve), 0.8 (green dashed curve), and 0.6 (black solid curve), respectively.

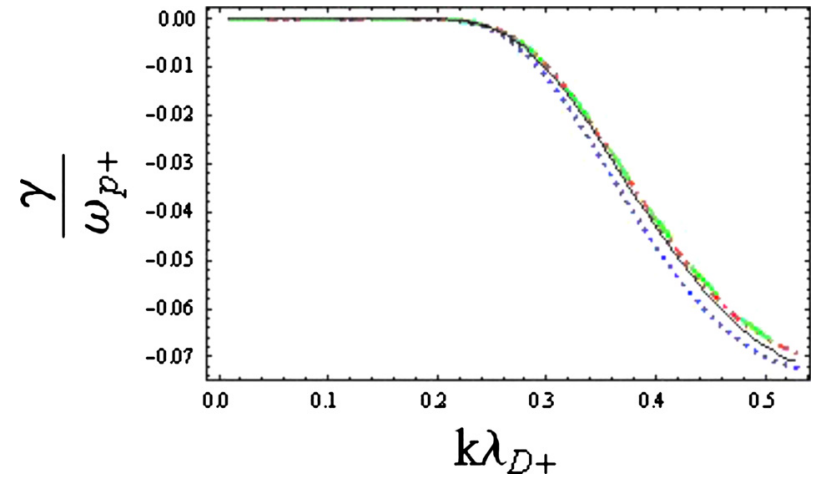

FIG. 5. (Color online) Normalized damping rates of electrostatic ion waves are shown for Maxwellian (i.e., $\kappa=50$ ) PI plasma for the case $T_{+} \geq T_{-}$, where damping curves correspond to $T_{+} / T_{-}=1$ (blue dotted curve), 1.2 (red dotdashed curve), 1.4 (green dashed curve), and 1.6 (black solid curve), respectively.

the pair-ion species. The Vlasov equation is solved for electrostatic ion waves by considering the generalized kappa distribution function for the pair-ion fullerene plasmas. The damping rates of the longitudinal waves are found to be larger in Lorentzian plasmas in comparison with the Maxwellian plasmas. It has already been reported the ions are collected by ion exciter hole and therefore the nonMaxwellian distributed PI plasmas may exist in the laboratory experiments. ${ }^{14}$ Our findings are general and may be applicable to explain some aspects of PI plasma experiments.

The authors are thankful to an anonymous referee for his useful suggestions to improve the manuscript.

${ }^{1}$ W. Oohara and R. Hatakeyama, Phys. Rev. Lett. 91, 205005 (2003).

${ }^{2}$ W. Oohara, D. Date, and R. Hatakeyama, Phys. Rev. Lett. 95, 175003 (2005).

${ }^{3}$ W. Oohara, Y. Kuwabara, and R. Hatakeyama, Phys. Rev. E 75, 056403 (2007).

${ }^{4}$ A. Hasegawa and P. K. Shukla, Phys. Scr. 2005 (T116), 105 (2005).

${ }^{5}$ P. K. Shukla and M. Khan, Phys. Plasmas 12, 014504 (2005).

${ }^{6}$ W. M. Moslem and P. K. Shukla, Phys. Lett. A 362, 463 (2007).

${ }^{7}$ F. Verheest, Phys. Plasmas 13, 082301 (2006).

${ }^{8}$ A. E. Dubinov, I. D. Dubinova, and V. A. Gordienko, Phys. Plasmas 13, 082111 (2006).

${ }^{9}$ V. A. Gordienko and A. E. Dubinov, High Temp. 45, 740 (2007).

${ }^{10}$ S. Mahmood, H. Ur-Rehman, and H. Saleem, Phys. Scr. 80, 035502 (2009).

${ }^{11}$ S. Mahmood, S. Hussain, and A. M. Mirza, Phys. Plasmas 17, 034504 (2010).

${ }^{12}$ W. Masood, S. Mahmood, and N. Imtiaz, Phys. Plasmas 16, 122306 (2009).

${ }^{13}$ W. M. Moslem, I. Kourakis, and P. K. Shukla, Phys. Plasmas 14, 032107 (2007).

${ }^{14}$ J. Vranjes, D. Petrovic, B. P. Pandey, and S. Poedts, Phys. Plasmas 15, 072104 (2008)

${ }^{15}$ H. Schamel and A. Luque, New J. Phys. 7, 69 (2005).

${ }^{16} \mathrm{H}$. Saleem, Phys. Plasmas 14, 014505 (2007).

${ }^{17}$ J. Vranjes and S. Poedts, Plasma Sources Sci. Technol. 14, 485 (2005).

${ }^{18}$ T. K. Baluku and M. A. Hellberg, Phys. Plasmas 15, 123705 (2008).

${ }^{19}$ S. P. Christon, D. G. Mitchell, D. J. Williams, L. A. Frank, C. Y. Huang, and T. E. Eastman, J. Geophys. Res. 93, 2562 (1988).

${ }^{20}$ M. Maksimovic, V. Pierrard, and J. F. Lemaire, Astron. Astrophys. 324, 725 (1997)

${ }^{21}$ V. Pierrard, H. Lamy, and J. Lemarie, J. Geophys. Res. 109, A02118 (2004).

${ }^{22}$ S. Zaheer, G. Murtaza, and H. A. Shah, Phys. Plasmas 11, 2246 (2004).

${ }^{23}$ N. Rubab, N. V. Erkaev, and H. K. Biernat, Phys. Plasmas 16, 103704 (2009). 